\title{
Spectral Edge Frequency as a Simple Quantitative Measure of the Maturation of Electrocortical Activity
}

\author{
HAZEL H. SZETO \\ Department of Pharmacology, Cornell University Medical College, New York, New York 10021
}

\begin{abstract}
The feasibility of using spectral edge frequency (SEF) as a single quantitative measure of the maturation of electrocortical activity is demonstrated in a large number of fetal lambs studied throughout the 3rd trimester. A total of $\mathbf{4 3}$ electrocortical recordings obtained from 23 fetal lambs ranging from 116-140 d were subjected to power spectral analysis using the fast Fourier transform. Spectral edge frequency was calculated as the frequency below which $90 \%$ of the power resides. The SEF was found to be highly sensitive in identifying the transition between high-voltage slow-activity and low-voltage fast-activity electrocortical activity. By $125 \mathrm{~d}$, the distribution of SEF clearly showed two distinct populations, a low SEF with peak values ranging from 3-9 Hz; and a high SEF $\left(\mathrm{SEF}_{\mathrm{H}}\right)$ with values ranging from 13-23 Hz. Increase in gestational age was associated with a progressive increase in $\mathrm{SEF}_{\mathrm{H}}$, which can be described by the linear function (SEF = $0.53 G A-51.3)$. This progressive increase in $\mathrm{SEF}_{\mathrm{H}}$ reflects the gradual acquisition of faster frequencies. In contrast, low SEF declined with age before $130 \mathrm{~d}$, but then stabilized at $4 \mathrm{~Hz}$ throughout the rest of gestation. As a result, there was a gradual increase in the separation between $\mathrm{SEF}_{\mathrm{H}}$ and low SEF as a function of gestational age. (Pediatr Res 27: 289-292, 1990)
\end{abstract}

\section{Abbreviations}

ECoG, electrocorticogram

HVSA, high-voltage slow activity

LVFA, low-voltage fast activity

SEF, spectral edge frequency

FFT, fast fourier transform

$\mathrm{SEF}_{\mathrm{L}}$, low frequency peak of the SEF distribution

$\mathrm{SEF}_{\mathrm{H}}$, high frequency peak of the SEF distribution

GA, gestational age

The ECoG is often used as a measure of CNS function. Electrocortical activity can be detected in prenatal life and has been shown to undergo maturational changes throughout late gestation. These developmental changes include the progression from an immature waveform pattern of variable frequencies to the typical HVSA and LVFA patterns described by a number of investigators (1-6); changes in the relative incidence of these two

Received July 25, 1989; accepted November 15, 1989.

Correspondence and reprint requests Hazel H. Szeto, M.D., Ph.D., Department of Pharmacology, Cornell University Medical College, 1300 York Avenue, New York, NY 10021 .

Supported in part by National Institute on Drug Abuse Grants DA-2475-09 and DA-03722-03. H.H.S. is the recipient of Research Scientist Development Award DA-00100-06 from the National Institute on Drug Abuse (Alcohol, Drug Abuse and Mental Health Administration). predominant patterns $(2,6)$; as well as maturational changes in the waveform characteristics of the two predominant patterns $(5$, 6).

Fetal electrocortical activity can also be affected by a variety of pathophysiologic and pharmacologic conditions, such as acute hypoxia $(5,7,8)$, and maternal administration of opiates $(9,10)$ and ethanol (11). Such alterations may range from changes in the relative incidence of the normal ECoG patterns to the induction of an abnormal ECoG pattern. Most of these studies, however, have only addressed the effects of acute perturbation of the ECoG. There is currently no information on the effects of chronic hypoxia or repetitive drug exposure on the maturation of the fetal ECoG.

To assess such long term effects, it is necessary to have a simple quantitative measure of ECoG maturation. The ECoG is an extremely complex signal that can only be fully characterized by both amplitude and frequency. Although visual analysis will reveal amplitude changes, it does not permit assessment of changes in frequency components. Furthermore, amplitude of the signal is dependent on the distance between the two recording electrodes, thus making it very difficult to compare data between subjects. However, power spectral analysis will allow quantitative analysis of changes in frequency components. Comparison of a large number of power spectra can be rather difficult and generally involves dividing the spectral data into several different wavebands.

We have found that maturational changes in the fetal ECoG can be readily quantitated by a single spectral parameter, the SEF. The SEF was originally defined as the highest frequency at which a significant amount of power is present in the ECoG (12). This may be interpreted as $95 \%$ of the power (12) or $90 \%$ of the power as used in our analysis. The SEF essentially provides a measure of the overall frequency of the signal, and has been used very effectively in demonstrating dose-related slowing of the human EEG after the administration of anesthetic agents (13). Inasmuch as increase in gestational age has been shown to be associated with an increase in maximum frequencies detected in the $\beta$-band $(15-32 \mathrm{~Hz})(14)$, it was believed that the SEF could also be used to illustrate the enhancement in maximal frequencies as a function of age. The suitability of this spectral parameter to serve as a single measure of the maturation of ECoG has been demonstrated in this study using ECoG data obtained from a large number of fetal sheep recorded at different times throughout the 3rd trimester.

\section{MATERIALS AND METHODS}

Animal preparation. Twenty-three fetal lambs were surgically instrumented for chronic intrauterine recording of electrocortical activity between 110-118 d of gestation, in accordance with guidelines approved by the Institution for the Care and Use of Animals. Details of the surgical procedure have been described previously (9). Briefly, four stainless steel screws (size 0-80) were 


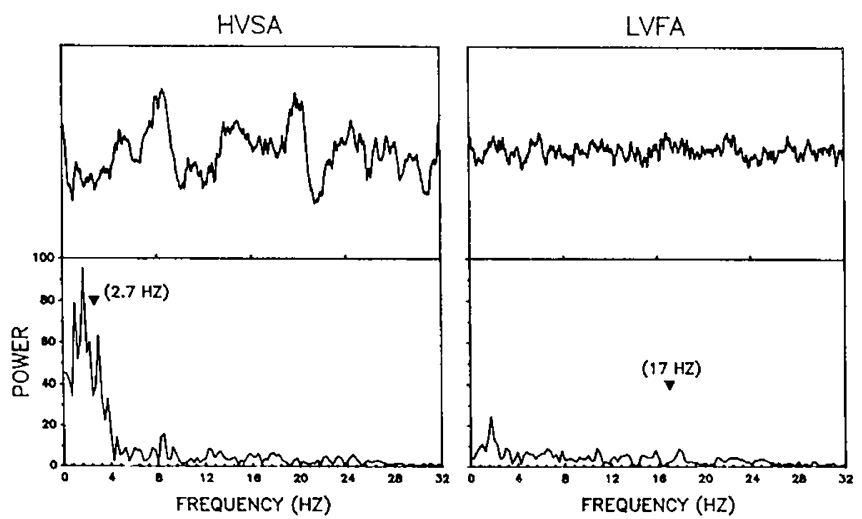

Fig. 1. Representative example of the analogue electrocorticogram record and power spectrum of an episode of HVSA and LVFA obtained in a 130-d fetal lamb. Top panel, analogue record; bottom panel, power spectrum. The spectral edge frequency is that frequency that has $90 \%$ of the area in the power spectrum to its left (represented by the solid triangle and the number in parentheses).

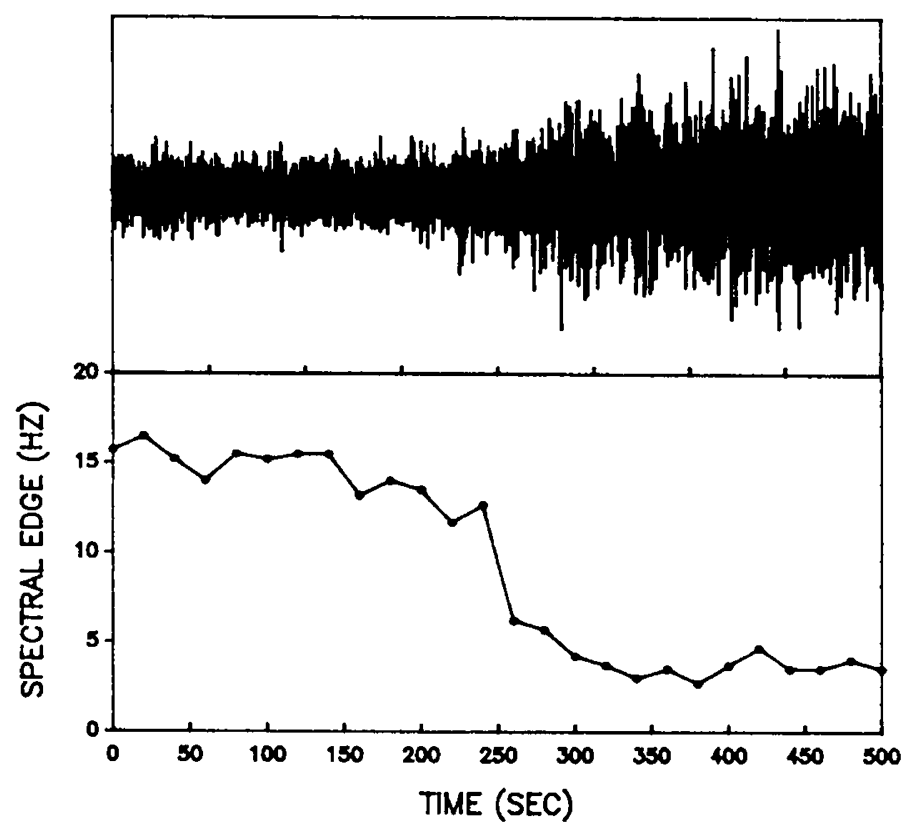

Fig. 2. Representative example of the analogue electrocorticogram record and the spectral edge frequency illustrating the transition from LVFA to HVSA in a 130-d fetal lamb. Top panel, analogue record (500 s); bottom panel, corresponding spectral edge frequency for the same ECoG segment. Each spectral edge value was calculated from the mean of 5 power spectra, taken $4 \mathrm{~s}$ apart.

implanted over the parietal cortex for recording ECoG activity. In addition, a polyvinyl catheter was placed in the distal aorta to permit collection of arterial blood for blood gas determinations. The ECoG leads and catheter were tunneled s.c. to the maternal flank and stored in a pouch. Intraoperatively, $2 \mathrm{~g}$ of ampicillin was placed in the amniotic cavity and $1 \mathrm{~g}$ in the peritoneal cavity of the ewe.

Recording procedures. Ewes were allowed at least $72 \mathrm{~h}$ after surgery before ECoG recording. Polygraphic recordings were obtained with the ewe standing or lying quietly in a cart. The ewe had free access to food and water throughout the recording period. The $1 \mathrm{st} h$ of recording was the habituation period and data recorded during this $1 \mathrm{st} h$ were excluded from the analysis. During this period a fetal arterial blood sample was collected for determination of arterial blood gases [Radiometer BMS3Mk2 blood gas analyzer (Radiometer America Inc., Westlake, OH), $37^{\circ} \mathrm{C}$ ] to assess fetal well being. Only fetuses with arterial $\mathrm{pH}>$

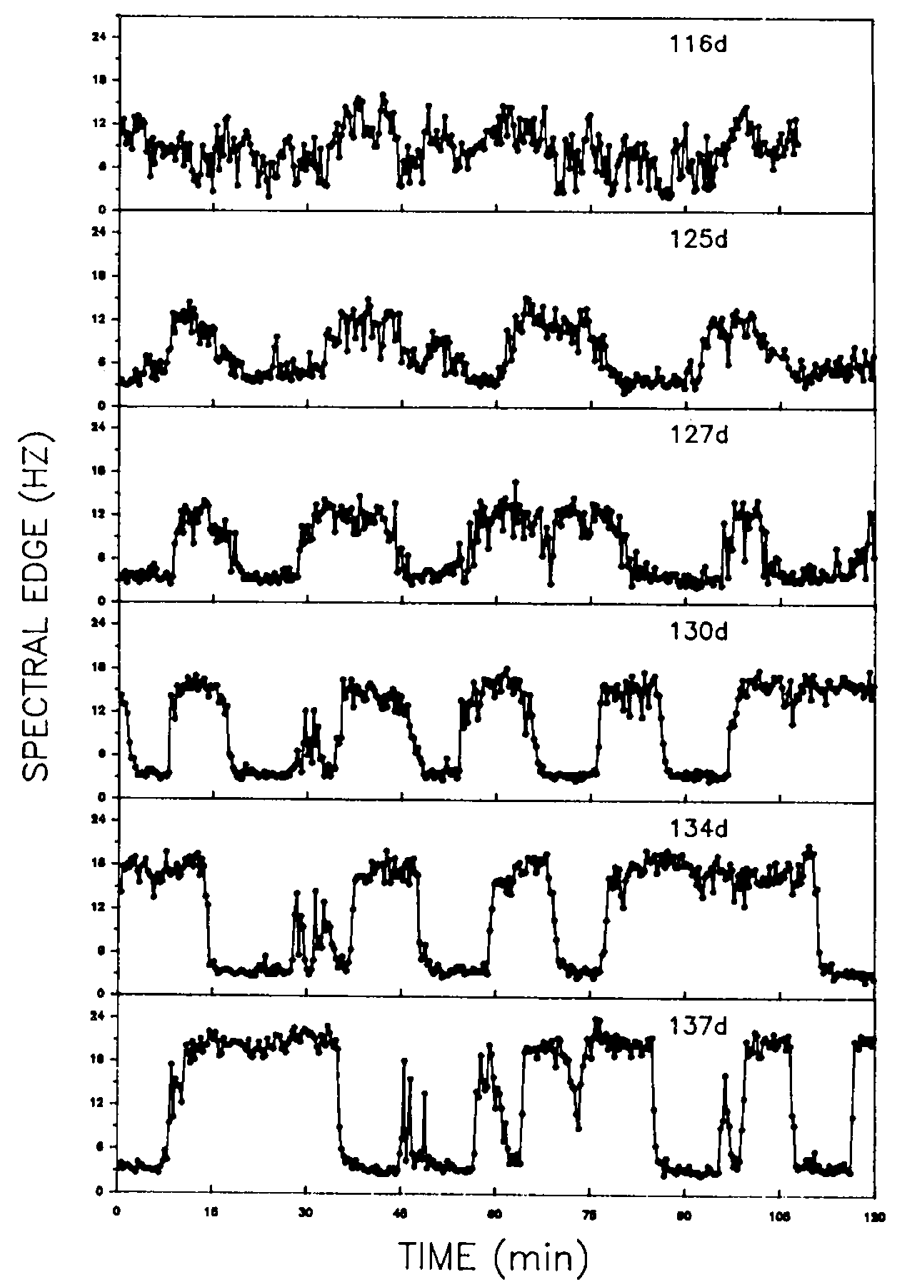

Fig. 3. Time series of spectral edge frequency obtained from the same fetal lamb on 6 different $\mathrm{d}$ from 116 to $137 \mathrm{~d}$. With the exception of the top panel $(116 \mathrm{~d})$, each panel represents $120 \mathrm{~min}$ of recording. Each spectral edge value was calculated from the mean of 5 power spectra, taken $4 \mathrm{~s}$ apart.

7.3, $\mathrm{PCO}_{2}<50 \mathrm{~mm} \mathrm{Hg}$ and $\mathrm{PO}_{2}>16 \mathrm{~mm} \mathrm{Hg}$ were included in the study. All recordings, which were $2-3 \mathrm{~h}$ in length, were obtained at the same time of day (0900 to $1200 \mathrm{~h})$.

Data acquisition and processing. The $\mathrm{ECoG}$ was recorded on a Gould 2800 S analogue recorder (Gould Inc., Santa Ana, CA) after amplification to about $1 \mathrm{~V}$ and with a bandpass filter of 1$100 \mathrm{~Hz}$. The amplified, filtered signal was also recorded concurrently onto FM tape (TEAC XR-310) for storage and off-line analysis. Analogue-to-digital conversion was accomplished with a board (DT-2801A, Data Translation, Marboro, MA) resident in an IBM AT microcomputer (IBM Instruments, Inc., Danbury, CT). Data were continuously digitized off-line at a rate of 256 $\mathrm{Hz}$, and stored in binary format.

Power spectral analysis. FFT was performed on consecutive series of 1024 detrended, windowed (Hamming) data points (4 s). Five power spectra were then averaged to produce a mean power spectrum. Averaging of spectra is necessary, as the error associated with each point estimate in a single power spectrum is $100 \%$; the mean of $\mathrm{N}$ runs of data produces an error term which is reduced to $\left.1 /(\mathrm{N}-1)^{1 / 2}\right)(15)$. The resolution of the FFT was $0.25 \mathrm{~Hz}$. The maximum detectable frequency was designated to be $64 \mathrm{~Hz}$, although data for frequencies more than $32 \mathrm{~Hz}$ were discarded. The spectral edge frequency was determined for each averaged spectrum as the frequency below which $90 \%$ of the power reside. 


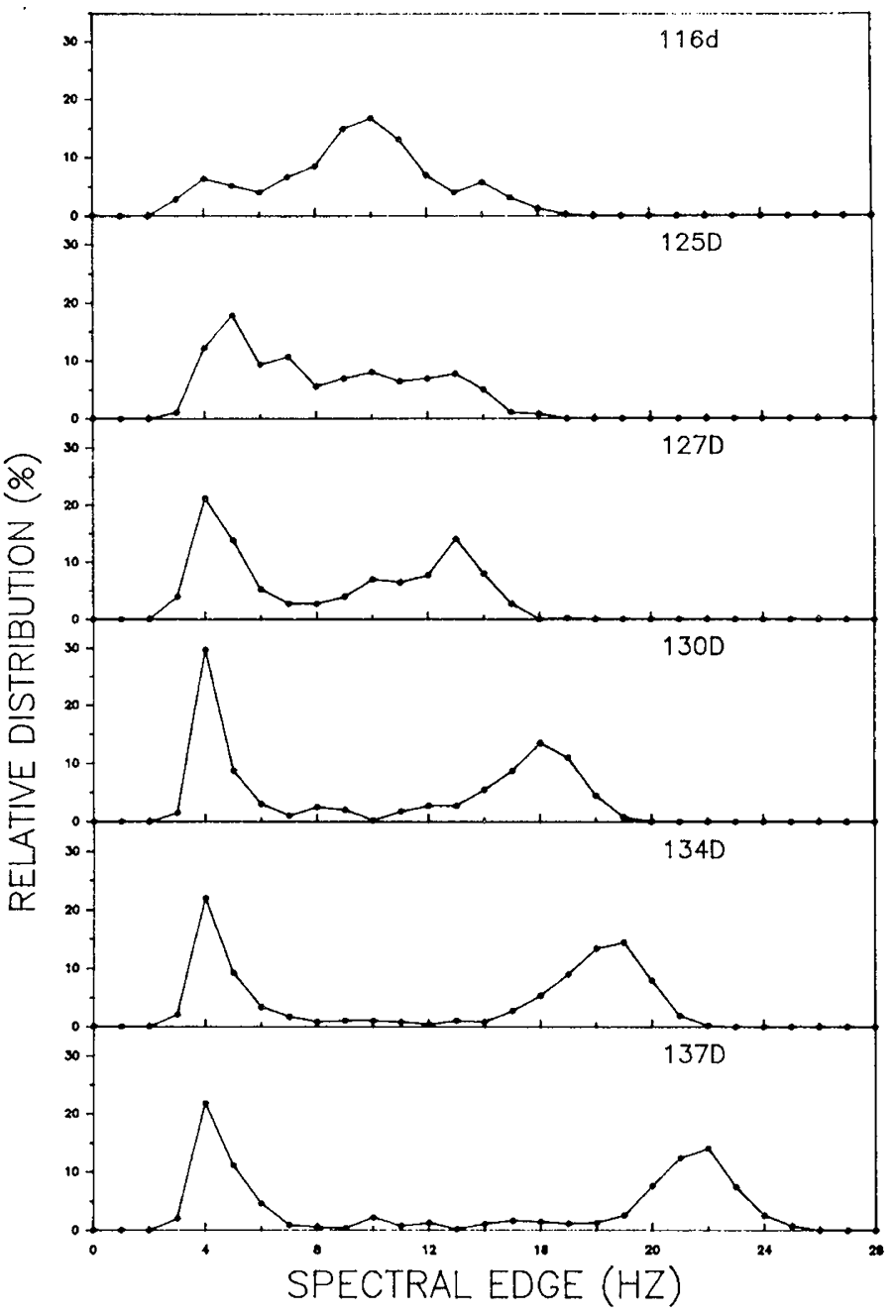

Fig. 4. Histograms illustrating the distribution of spectral edge values obtained on the 6 different $d$ from the same fetal lamb as shown in Figure 3. With the exception of the top panel (116 d), each histogram represents the distribution of a total of 360 spectral edge values.

\section{RESULTS}

A total of $42 \mathrm{ECoG}$ recordings were obtained from 23 fetal lambs ranging from 116-140 d of gestation. These were normal undisturbed animals that had not been subjected to any experimental procedures before data acquisition. Eight of these animals were studied on two to six different occasions. The other 15 animals were only studied once for this purpose and were then used for other studies. The data were grouped as follows, according to gestational age of the fetus: $116-120 \mathrm{~d}, 121-125 \mathrm{~d}, 126-$ $130 \mathrm{~d}, 131-135 \mathrm{~d}$, and 136-140 d. The number of animals in each age group ranged from 6-10.

Spectral edge frequency was found to be highly effective in differentiating between HVSA and LVFA in mature fetal lambs. Figure 1 illustrates the difference in SEF in a 130-d fetal lamb during HVSA and LVFA, respectively. The solid triangle and the number in parentheses indicate the frequency below which $90 \%$ of the power resides (SEF).

The $90 \%$ SEF was found to be an excellent index of cyclic ECoG activity. A representative polygraphic record, as well as the corresponding SEF time series, are shown in Figure 2. The change from LVFA to HVSA is reflected by a gradual decrease in the SEF.

Figure 3 illustrates the SEF time series (120 min) obtained on 6 different $d$ from the same fetal lamb. The distribution of the SEF values (360 runs) on the various days is illustrated as a histogram in Figure 4. Although cyclic fluctuation was seen in

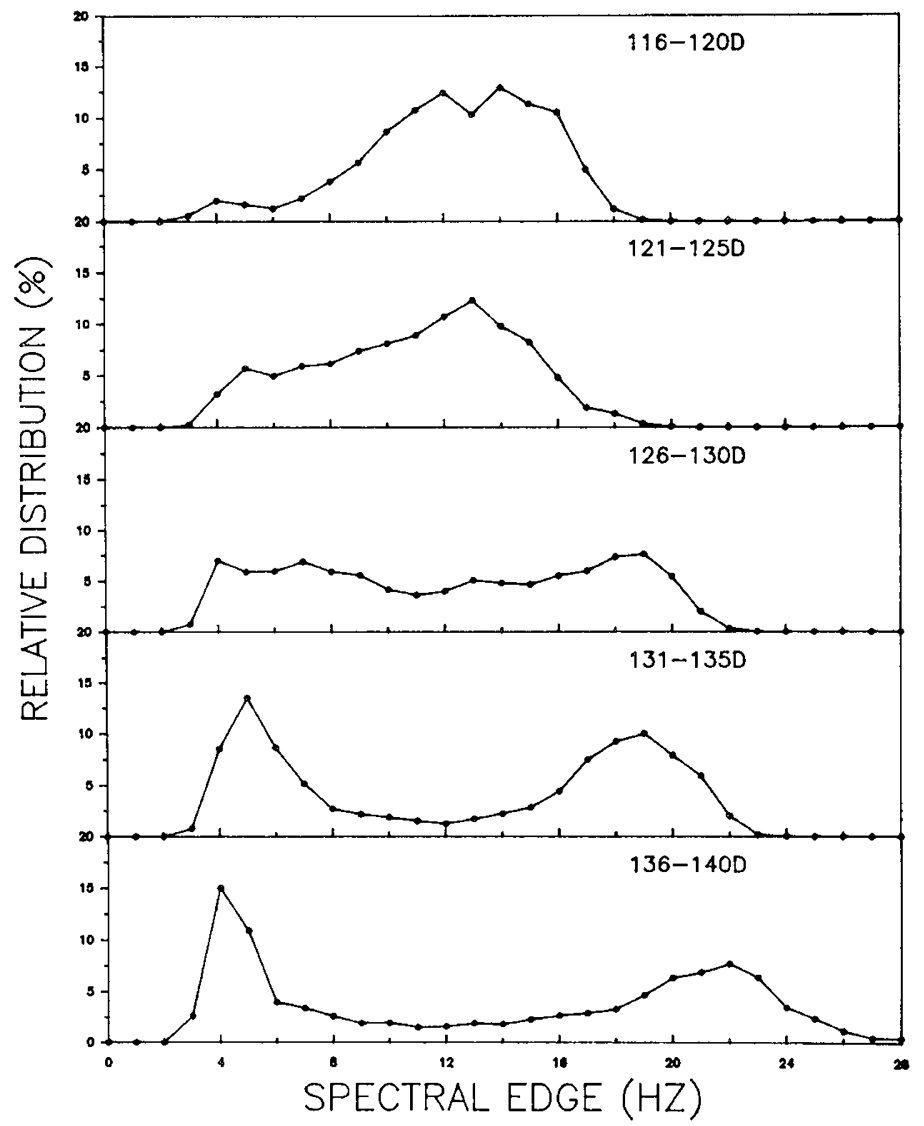

Fig. 5. Histograms illustrating the mean distribution of spectral edge values in five gestational age groups: $116-120 \mathrm{~d} ; 121-125 \mathrm{~d} ; 126-130 \mathrm{~d}$; 131-135 d; and 136-140 d. Each gestational age group represents six to 10 animals, with 360 spectral edge values obtained from each animal.

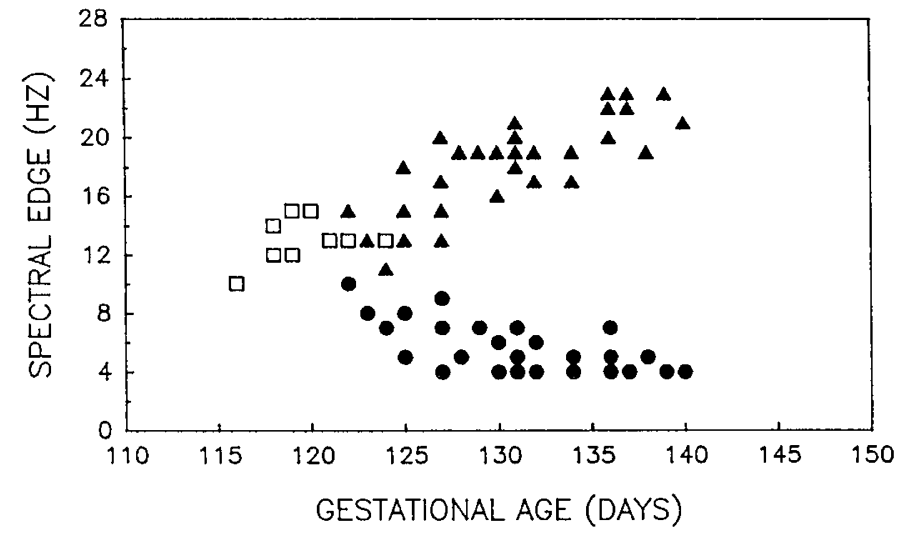

Fig. 6. Growth curve showing the change in the dominant frequency in the spectral edge distribution as a function of gestational age in individual animals. The open squares indicate the dominant frequency (the mode of the distribution) in cases where only one peak can be identified. The solid circles represent the dominant frequency of the high frequency peak, whereas the solid triangles represent the dominant frequency of the low frequency peak. This curve encompasses data from all 43 recordings. Overlapping data are not illustrated.

the SEF over a 120-min interval at $116 \mathrm{~d}$, there was much variation in the data and little evidence of stable states. The corresponding histogram revealed a broad distribution with only a single peak at $10 \mathrm{~Hz}$. By $127 \mathrm{~d}$, stable states can be seen during which there is little variation in the SEF from run to run, and two distinctly separated peaks can be identified in the histogram. The peak with $\mathrm{SEF}_{\mathrm{L}}$ values corresponds to the HVSA episodes, whereas the peak with $\mathrm{SEF}_{\mathrm{H}}$ values corresponds to the LVFA 
episodes. From 127 to $137 \mathrm{~d}$, there was a progressive increase in $\mathrm{SEF}_{\mathrm{H}}$ resulting in a gradual increase in the separation of the two peaks. There was little change in the distribution of the $\mathrm{SEF}_{\mathrm{L}}$ episodes between 127 to $137 \mathrm{~d}$.

Figure 5 represents the distribution histogram of SEF obtained from all the animals. A total of six to 10 animals was studied at each age group. As in the data obtained from the individual animal (Figs. 3 and 4), there was a progressive separation of the two peaks with advancing gestational age. However, the maturation of spectral edge appears to be slower in the combined data, with the separation of the two peaks not becoming evident until 126-130 d. This was due to interindividual variation in the rate of maturation of the two stable states, as well as variation in the dominant frequencies.

The interindividual variation is illustrated in Figure 6 where the dominant frequencies are plotted as a function of gestational age for all animals. The open squares indicate the dominant frequency (the mode of the distribution) in cases where only one peak can be identified. The solid circles represent the dominant frequency of the $\mathrm{SEF}_{\mathrm{H}}$, whereas the open triangles represent the dominant frequency of the $\mathrm{SEF}_{\mathrm{L}}$. The earliest identification of the two peaks was observed in a fetus at $122 \mathrm{~d}$. However, two distinct peaks were seen in all animals by $125 \mathrm{~d}$. From that time on, there was a linear increase in the $\mathrm{SEF}_{\mathrm{H}}$ as a function of age, which can be described by the following mathematical function:

$$
\mathrm{SEF}_{\mathrm{H}}=0.53 \mathrm{GA}-51.3(r=0.82)
$$

In contrast, there was a decline in $\mathrm{SEF}_{\mathrm{L}}$ before $130 \mathrm{~d}$, after which it stabilized at approximately $4 \mathrm{~Hz}$ throughout the rest of gestation.

\section{DISCUSSION}

We have previously demonstrated that although cycling patterns in ECoG activity can be visually identified by as early as $115 \mathrm{~d}$ gestation in the fetal lamb, quantitative changes in waveform characteristics continue throughout the rest of gestation (14). These maturational changes were found to occur primarily in the LVFA episodes, with no significant changes in the power spectrum of the HVSA episodes. Increase in gestational age was also associated with a decrease in power density in the $\delta$-band $(1-4 \mathrm{~Hz})$ and an increase in power density in the $\beta$-band $(15-32$ $\mathrm{Hz}$ ). This reflected an increase in the overall frequency of the signal.

The results of this study are in agreement with our previous findings. The SEF was very effective in demonstrating the onset of cyclic behavior in the ECoG. Although cyclic variations were seen in the SEF as early as $116 \mathrm{~d}$, the two stable states were not clearly established until 122-125 d. Although visual analysis of the ECoG suggested that two dominant patterns could be distinguished as early as $115 \mathrm{~d}$, the SEF revealed that these early patterns are not as homogenous as had been thought.

After the onset of the two stable states of ECoG, there was subsequently a linear increase in the SEF during LVFA $\left(\mathrm{SEF}_{\mathrm{H}}\right)$, implying an increase in faster frequencies with age. There was also a decrease in the SEF during HVSA $\left(\mathrm{SEF}_{\mathrm{L}}\right)$ from 125-130 $\mathrm{d}$, indicating the acquisition of slower frequencies. However, there was little change in $\mathrm{SEF}_{\mathrm{L}}$ after $130 \mathrm{~d}$. This is in agreement with our previous finding that there were no significant changes in the power spectrum of the HVSA episodes.

These results illustrate how a SEF can be used to encompass many of the maturational changes in ECoG that were described previously (14). Spectral edge frequency is simple to compute, and provides a method for the reduction of large volumes of spectral data. Furthermore, the maturational changes in SEF can be described mathematically, permitting quantitative comparisons to be made. Long-term alterations in ECoG may be represented as a shift in this developmental curve and/or a change in the slope of the curve. Inasmuch as the curve describes the SEF of both HVSA and LVFA, it also permits adverse effects on the two states to be differentiated.

The SEF will also be useful in quantitating the effects on ECoG after acute pathophysiologic or pharmacologic perturbations. At the present time, these changes have mostly been quantitated by visual analysis, and it is not known whether the frequency characteristics have been altered. Statistical comparison of the distribution histograms before and after perturbation will permit not only an assessment of whether the frequency components have been affected, but also whether the relative distribution of the two physiologic patterns have been changed. In addition, a number of perturbations have been reported to induce a pattern of "intermediate voltage" and previously indeterminate frequency. The SEF would enable quantitative assessment of frequency as well.

\section{REFERENCES}

1. Mann LI 1970 Fetal brain metabolism and function. Clin Obstet Gynecol 13:638-651

2. Ruckebusch $Y 1972$ Development of sleep and wakefulness in the foetal lamb. Electroencephalogr Clin Neurophysiol 32:119-128

3. Dawes GS, Fox HE, Leduc BM, Liggins GC, Richards RT 1972 Respiratory movements and rapid eye movement sleep in the foetal lamb. $J$ Physiol (Lond) 220:119-143

4. Ruckebusch Y, Gaujoux M, Eghbali B 1977 Sleep cycles and kinesis in the foetal lamb. Electroencephalogr Clin Neurophysiol 42:226-237

5. Clelow F, Dawes GS, Johnston BM, Walker DW 1983 Changes in breathing, electrocortical activity in unanaesthetized fetal lambs with age. J Physiol (Lond) 341:463-476

6. Szeto HH, Hinman DJ 1985 Prenatal development of sleep-wake patterns in sheep. Sleep 8:347-355

7. Symmes D, Prichard JW, Mann LI 1970 Spectral analysis of fetal sheep EEG during hypoxia. Electroencephalogr Clin Neurophysiol 29:511-515

8. Boddy K, Dawes GS, Fisher R, Pinter S, Robinson JS 1984 Foetal respiratory movements, electrocortical and cardiovascular responses to hypoxia and hypercapnia in sheep. J Physiol (Lond) 243:599-618

9. Szeto HH 1983 Effects of narcotic drugs on fetal behavioral activity: acute methadone exposure. Am J Obstet Gynecol 146:211-217

10. Umans JG, Szeto HH 1983 Effects of opiates on fetal behavioral activity in utero. Life Sci 33:639-642

11. Patrick J, Carmichael L, Richardson B, Smith G, Homan J, Brien J 1988 Effects of multiple-dose maternal ethanol infusion on fetal cardiovascular and brain activity in lambs. Am J Obstet Gynecol 159:1424-1429

12. Rampil IJ, Sasse FJ, Smith NT, Hoff BH, Fleming DC 1980 Spectral edge frequency-a new correlate of anesthetic depth. Anesthesiology 53:S12.

13. Scott JC, Ponganis KV, Stanski DR 1985 EEG quantitation of narcotic effect: The comparative pharmacodynamics of fentanyl and alfentanil. Anethesiology $62: 234-241$

14. Szeto HH, Vo TDH, Dwyer G, Dogramajian ME, Cox M, Senger G 1985 The ontogeny of fetal lamb electrocortical activity: a power spectral analysis. Am J Obstet Gynecol 153:462-466

15. Bendat JS, Piersol AG 1986 Random Data-Analysis and Measurement Procedures, 2nd ed. John Wiley \& Sons, New York, pp 263-268 